\title{
Cause of postoperative mortality in patients with end-stage renal disease
}

\section{Sanghoon Song, Chaeyeon Cho, Sun Young Park, Ho Bum Cho, Jae Hwa Yoo, Mun Gyu Kim, Ji Won Chung, and Sang Ho Kim}

Department of Anesthesiology and Pain Medicine, Soonchunhyang University Seoul Hospital, Seoul, Korea

\begin{abstract}
Background: The number of patients with end-stage renal disease (ESRD) who are dependent on hemodialysis is increasing rapidly. As a result, more patients with ESRD need surgery. These patients have a significantly higher risk of postoperative death than those with normal kidney function. Therefore, this study analyzed the causes of postoperative mortality in ESRD patients undergoing surgery under general anesthesia and the risk factors for postoperative mortality.
\end{abstract}

Methods: This retrospective analysis examined the mortality of ESRD patients, 20 to 80 years old, undergoing surgery under general anesthesia. We excluded patients who underwent cardiac, cancer, or emergency surgery or organ transplantation from the analysis. The primary outcome was the cause of postoperative 30-day mortality in ESRD patients. We also assessed the mortality rate and risk factors.

Results: There were 2,459 eligible ESRD patients. When patients underwent multiple surgeries during the study period, only the last surgery was considered. In total, 167 patients died during the study period, including 65 within 30 days postoperatively. The cause of death was sepsis in 22 cases (33.8\%) and a major cardiac event in 16 (24.6\%). Atrial fibrillation, current angina, previous myocardial infarction, asthma, lower hemoglobin and albumin levels, and a larger intraoperative colloid volume were likely to increase mortality.

Conclusions: Our study suggests that immunological issues have a significant role in the death of ESRD patients after general anesthesia.

Keywords: Cause of death; End-stage renal disease; General anesthesia; Infection.

\section{INTRODUCTION}

End-stage renal disease (ESRD) is chronic kidney disease (CKD) stage 5 and these patients need lifelong dialysis or transplantation for survival $[1,2]$. CKD patients tend to be older ( $>65$ years) and have diabetes mellitus (DM), hypertension, coronary vessel disease, and obesity [3-5]. The prevalence of ESRD in the USA has almost doubled in the last 20 years due to an increasing number of incident cases and the prolonged survival of ESRD patients, supposedly due to improved hemodialysis (HD) care [2,6]. In Korea, there were more than 100,000 ESRD patients in 2019, and the number is increasing by $7-10 \%$ annually [7]. The number of patients with ESRD who need surgery has increased with the number of HD-dependent patients with ESRD $[2,8]$. Moreover, patients with ESRD have a significantly higher risk of postoperative death than those with normal kidney function $[2,9-12]$.

This is an Open Access article distributed under the terms of the Creative Commons Attribution Non-Commercial License (http://creativecommons.org/licenses/by-nc/4.0) which permits unrestricted non-commercial use, distribution, and reproduction in any medium, provided the original work is properly cited.

Copyright (C) the Korean Society of Anesthesiologists, 2022 
In non-cardiac surgery, patients with CKD had a two- to five-fold higher risk of cardiovascular events than those with normal kidney function [13]. Patients with ESRD undergoing HD were nearly three times as likely to return to the operating room, and their postoperative 30-day mortality was ten times higher than those without ESRD $[11,12]$. Therefore, anesthesiologists and surgeons have to pay particular attention to these high-risk patients. We conducted this study to analyze the primary cause of postoperative 30-day mortality in patients with ESRD undergoing surgery under general anesthesia. We also evaluated potential risk factors for postoperative 30-day mortality in these patients.

\section{MATERIALS AND METHODS}

This retrospective single-center study ran from January 2018 to January 2020. This retrospective single-center study ran from January 2018 to January 2020. The Institutional Review Board of Soonchunhyang University Hospital, Seoul, Republic of Korea, approved this study (no. SCHUH 2021-07015). Patient demographics, comorbidities, and laboratory results were obtained from the hospital's electronic medical records. An initial search identified all patients 20 to 80 years old with ESRD who underwent general anesthesia. The patients who underwent cardiac, cancer, or emergency surgery or organ transplantation were excluded. General anesthesia combined with any type of regional block was included. When patients underwent multiple surgeries during the study period, only the last surgery was eligible. The requirement for informed consent was waived because of the study design.

The primary outcome was the primary cause of postoperative 30-day mortality in ESRD patients. The cause of death was defined as that recorded in the electronic medical records or registered in national data. The secondary outcomes were the all-cause postoperative 30-day mortality rate in ESRD patients and the identified risk factors for postoperative 30-day mortality. Mortality data, including date and primary cause of death, were obtained from the electronic medical records and by submitting data requests to the Korean National Statistical Office (Microdata Integrated Service, on-demand, https://mdis.kostat.go.kr). If the cause of death was recorded as 'ESRD', it was classified as 'Unknown'. Risk factors were identified statistically among demographic, comorbidity, and preoperative laboratory parameters. In our institution, when a patient diagnosed with ESRD or requiring dialysis is scheduled for surgery, we check the blood urea nitrate, creatinine, and electrolytes (sodium, potassium, and chloride) after preoperative dialysis and early the morning of surgery.

\section{Statistical analysis}

Multivariable logistic regression analysis was performed to identify risk factors for postoperative 30-day mortality under general anesthesia. The factors included as potential confounding variables were age, sex, body mass index (BMI), relevant pre-admission comorbidities subsequent atrial fibrillation, current angina pectoris, congestive heart failure, valvular heart disease, dilated cardiomyopathy, asthma, chronic obstructive pulmonary disease, interstitial lung disease, liver cirrhosis, diabetes mellitus, cerebrovascular disease, dementia, hypertension, previous myocardial infarction, preoperative post HD sodium, potassium, chloride, hemoglobin, albumin, and calcium, type and duration of anesthesia, and total amounts of crystalloid and colloid administered. The relations between mortality and potential confounding variables were evaluated separately in a univariable logistic model and those with $\mathrm{P}<0.1$ were subsequently subject to multivariable logistic regression. All variables included in the multivariable model required complete patient data; cases with missing co-variates were excluded from the analysis. We used a two-sided 5\% a-level to evaluate differences in the model. We report the cause and mortality rate as a number and percentage and the odds ratios (OR) of confounding variables for postoperative 30-day mortality along with the $95 \%$ confidence intervals. Values of $\mathrm{P}<0.05$ were considered statistically significant. All statistical analyses were performed using Rex Excel-based statistical analysis software, ver. 3.6.0 (RexSoft, Korea, http://rexsoft.org/) based on R ver. 4.0.0 (R Foundation for Statistical Computing, Austria).

\section{RESULTS}

Initially, 3,367 surgical procedures in ESRD patients were extracted from our hospital database. After excluding 31 organ transplantations, 13 cardiac surgeries, 50 cancer surgeries, 180 emergency surgeries, and 634 additional procedures performed in the same patients, 2,469 patients were included in the study. Table 1 summarizes the baseline characteristics of the study population. Of the 2,459 patients, 2,029 $(82.5 \%)$ were diagnosed with hypertension preoperatively, and 1,223 (49.7\%), 361 (14.7\%), and 306 (12.4\%) were diagnosed with diabetes mellitus, cerebrovascular disease, and 
Table 1. Baseline Characteristics of the Patients with ESRD Undergoing General Anesthesia

\begin{tabular}{|c|c|}
\hline Characteristics & Total $(n=2,459)$ \\
\hline \multicolumn{2}{|l|}{ Patient factor } \\
\hline Age (yr) & $64.9 \pm 41.3$ \\
\hline Sex, male & $551(53.0)$ \\
\hline Weight (kg) & $61.3 \pm 20.4$ \\
\hline Height (cm) & $160.8 \pm 9.9$ \\
\hline $\mathrm{BMI}\left(\mathrm{kg} / \mathrm{m}^{2}\right)$ & $23.5 \pm 5.0$ \\
\hline Atrial fibrillation & $155(6.3)$ \\
\hline Current angina pectoris & $144(5.9)$ \\
\hline Previous Ml & $101(4.1)$ \\
\hline Congestive heart failure & $65(2.6)$ \\
\hline Valvular heart disease & $306(12.4)$ \\
\hline Dilated cardiomyopathy & $2(0.1)$ \\
\hline $\mathrm{TIA} / \mathrm{CVA}$ & $361(14.7)$ \\
\hline Hypertension & $2,029(82.5)$ \\
\hline Diabetes mellitus & $1,223(49.7)$ \\
\hline Asthma & $49(2.0)$ \\
\hline COPD & $25(1.0)$ \\
\hline ILD & $1(0.04)$ \\
\hline Post HD plasma $\mathrm{Na}$ (mmol/L) & $140.3 \pm 7.2$ \\
\hline Post HD plasma K (mmol/L) & $4.4 \pm 0.7$ \\
\hline Post HD plasma $\mathrm{Cl}$ (mmol/L) & $100.5 \pm 5.2$ \\
\hline Post HD plasma BUN (mg/dl) & $34.9 \pm 22.4$ \\
\hline Post HD plasma $\mathrm{Cr}$ (mg/dl) & $5.4 \pm 3.3$ \\
\hline Hemoglobin (g/dl) & $11.2 \pm 1.7$ \\
\hline Hematocrit (\%) & $34.4 \pm 5.2$ \\
\hline Platelet $\left(10^{3} / \mu \mathrm{l}\right)$ & $192.0 \pm 74.1$ \\
\hline Plasma albumin (g/dl) & $4.2 \pm 1.0$ \\
\hline Plasma Ca (mg/dl) & $9.2 \pm 2.3$ \\
\hline \multirow[t]{2}{*}{ Ejection Fraction (\%) } & $63(58,69)$ \\
\hline & $n=1,401(57 \%)$ \\
\hline \multicolumn{2}{|l|}{ Perioperative factor } \\
\hline Volatile anesthesia & $1,227(49.9)$ \\
\hline Intraoperative crystalloids (ml) & $226.5 \pm 355.5$ \\
\hline Intraoperative colloids (ml) & $28.8 \pm 110.4$ \\
\hline Anesthetic duration (min) & $126.0 \pm 64.5$ \\
\hline
\end{tabular}

Values are presented as mean $\pm \mathrm{SD}$, number of patients (\%), or median (1Q, 3Q). ESRD: end-stage renal disease, BMI: body mass index, MI: myocardial infarction, TIA: transient ischemic attack, CVA: cerebrovascular disease, COPD: chronic obstructive pulmonary disease, ILD: interstitial lung disease, HD: hemodialysis, BUN: blood urea nitrogen, Cr: creatinine.

valvular heart disease, respectively.

Within this study period, 167 patients $(6.8 \%)$ died, of whom 65 (2.6\%) died within 30 days postoperatively. The most common cause of death was sepsis (22 patients, 33.8\%) followed by major cardiac events (16 patients, 24.6\%) (Table 2). Major cardiac events included myocardial infarction (ST-elevation,
Table 2. Primary Causes of Postoperative 30-day Mortality in Patients with End-stage Renal Disease

\begin{tabular}{lc}
\hline \multicolumn{1}{c}{ Cause of death } & Total $(\mathrm{n}=65)$ \\
\hline Sepsis & $22(33.8)$ \\
Major cardiac event & $16(24.6)$ \\
Pneumonia & $4(6.2)$ \\
Thromboembolic event & $4(6.2)$ \\
Cancer & $3(4.6)$ \\
Diabetes mellitus & $2(3.1)$ \\
Hypovolemia & $2(3.1)$ \\
Cerebral infarction & $2(3.1)$ \\
Liver cirrhosis & $1(1.5)$ \\
Bleeding & $1(1.5)$ \\
Unknown & $8(12.3)$ \\
\hline
\end{tabular}

Values are presented as number of patients (\%). Data were obtained from electronic medical records and by submitting a data request to the Korean National Statistical Office (Microdata Integrated Service, on-demand, https://mdis.kostat.go.kr). 'Major cardiac event' comprised ST-elevation, non ST-elevation, and acute myocardial infarctions, ischemic heart disease, aortic stenosis, congestive heart failure, secondary pulmonary hypertension, and cardiac arrest. If the cause of death was written as 'ESRD', it was classified as 'Unknown'.

non ST-elevation, and acute), ischemic heart disease, aortic stenosis, congestive heart failure, secondary pulmonary hypertension, and cardiac arrest. The category 'Unknown' contained eight patients (12.4\%) with out-of-hospital deaths of unknown cause or 'ESRD'. Other causes of death were pneumonia (four patients, $6.2 \%$ ), thromboembolic event (four patients, 6.2\%), and cancer (three patients, 4.6\%). For the patients whose cause of death was categorized as 'sepsis', along with a statement in the medical records, we found evidence for the diagnosis based on 'The Third International Consensus Definitions for Sepsis and Septic Shock, JAMA, 2016' [14]. Regarding the origin of sepsis, no case was directly related to surgical site infection in our study. If 'pneumonia' was the primary cause of sepsis, the case was categorized as 'pneumonia'

The multivariable analysis including 15 factors with $\mathrm{P}<$ 0.1 in the univariable analyses identified atrial fibrillation, previous myocardial infarction, asthma, hemoglobin, plasma albumin, volatile anesthesia, and intraoperative colloid volume as statistically significant (Table 3). All patients were included in the analysis because there were no missing data.

\section{DISCUSSION}

In our study, the most common cause of death was sepsis (33.8\%), followed by major cardiac events $(24.6 \%)$, suggest- 
Table 3. Expected Risk Factors as Odds Ratio for Postoperative 30-day Mortality in Patients with End-stage Renal Disease according to the Baseline Characteristics

\begin{tabular}{|c|c|c|c|c|}
\hline \multirow{2}{*}{ Variables } & \multicolumn{2}{|c|}{ Univariable analysis } & \multicolumn{2}{|c|}{ Multivariable analysis } \\
\hline & Crude OR (95\% Cl) & $P$ value & Adjusted OR (95\% Cl) & $P$ value \\
\hline \multicolumn{5}{|l|}{ Patient factor } \\
\hline Age (yr) & $1.00(1.00-1.00)$ & 0.243 & & \\
\hline Sex, male & $0.94(0.66-1.34)$ & 0.726 & & \\
\hline BMI $\left(\mathrm{kg} / \mathrm{m}^{2}\right)$ & $0.92(0.86-0.99)$ & 0.020 & $0.94(0.88-1.02)$ & 0.128 \\
\hline Atrial fibrillation & $2.00(1.15-3.49)$ & 0.015 & $2.14(1.21-3.78)$ & 0.009 \\
\hline Current angina pectoris & $2.05(0.96-4.40)$ & 0.065 & $1.13(0.47-2.70)$ & 0.790 \\
\hline Previous MI & $4.46(2.04-7.94)$ & $<0.001$ & $2.90(1.12-7.59)$ & 0.029 \\
\hline Congestive heart failure & $1.24(0.50-3.50)$ & 0.645 & & \\
\hline Valvular heart disease & $3.67(2.13-6.34)$ & $<0.001$ & $1.80(0.90-3.60)$ & 0.096 \\
\hline Dilated cardiomyopathy & $1.844 \times 10^{-05}(0$-inf $)$ & 0.978 & & \\
\hline TIA/CVA & $1.48(0.79-2.77)$ & 0.224 & & \\
\hline Hypertension & $0.71(0.47-1.08)$ & 0.109 & & \\
\hline Diabetes mellitus & $1.04(0.73-1.48)$ & 0.848 & & \\
\hline Asthma & 5.87 (2.39-14.39) & 0.001 & $7.72(2.79-21.38)$ & $<0.001$ \\
\hline COPD & $1.29(0.31-5.39)$ & 0.728 & & \\
\hline ILD & $39.54(0.45-637.61)$ & 0.985 & & \\
\hline Dementia & $4.998(2.18-11.47)$ & $<0.001$ & $2.62(0.99-6.92)$ & 0.052 \\
\hline Hemoglobin (g/dl) & $0.60(0.51-0.70)$ & $<0.001$ & $0.57(0.43-0.76)$ & $<0.001$ \\
\hline Post HD plasma $\mathrm{Na}(\mathrm{mmol} / \mathrm{L})$ & $0.99(0.97-1.01)$ & 0.502 & & \\
\hline Post HD plasma K (mmol/L) & $0.66(0.47-0.84)$ & 0.002 & & \\
\hline Hyperkalemia ( > $5.1 \mathrm{mmol} / \mathrm{L}$ ) & $1.44(0.59-3.52)$ & 0.430 & & \\
\hline Hypokalemia (< 3.5 mmol/L) & $3.73(1.89-7.36)$ & $<0.001$ & $1.78(0.81-3.93)$ & 0.152 \\
\hline Post HD plasma Cl (mmol/L) & $1.00(0.96-1.04)$ & 0.950 & & \\
\hline Plasma albumin (g/dl) & $0.22(0.15-0.31)$ & $<0.001$ & $0.35(0.22-0.56)$ & $<0.001$ \\
\hline Plasma Ca (md/dl) & $0.67(0.50-0.90)$ & 0.009 & $1.09(0.75-1.60)$ & 0.647 \\
\hline \multicolumn{5}{|l|}{ Perioperative factor } \\
\hline Volatile anesthesia & $3.08(1.07-8.82)$ & 0.036 & $3.21(1.10-9.34)$ & 0.032 \\
\hline Anesthesia duration (min) & $1.01(1.00-1.01)$ & $<0.001$ & $1.00(1.00-1.01)$ & 0.281 \\
\hline Intraoperative crystalloids (ml) & $1.00(1.00-1.00)$ & $<0.001$ & $1.00(1.00-1.00)$ & 0.950 \\
\hline Intraoperative colloids (ml) & $1.00(1.00-1.00)$ & $<0.001$ & $1.00(1.00-1.00)$ & 0.011 \\
\hline
\end{tabular}

OR: odds ratio, Cl: confidence interval, BMI: body mass index, MI: myocardial infarction, TIA: transient ischemic attack, CVA: cerebrovascular disease, COPD: chronic obstructive pulmonary disease, ILD: interstitial lung disease, HD: hemodialysis. Model Effect (Goodness of Fit Test) $P$ value $1.497 \times 10^{-15}$. Accuracy $=0.9747, A \cup C=0.7512$

ing that sepsis is the main cause of postoperative mortality in ESRD patients. The postoperative 30-day mortality of ESRD patients was $2.6 \%$.

As has been well established, the primary cause of morbidity and mortality in patients after non-cardiac surgery is that of adverse cardiovascular events [15]. CKD is a significant risk factor for perioperative adverse cardiovascular events because the presence of CKD may predispose patients to changes in the sodium and fluid balance, vascular calcification, and inflammatory changes, leading to atherosclerotic plaque destabilization [16]. Across various types of non-cardiac surgery, patients with ESRD had a significantly higher risk of postoperative death and cardiovascular events than those with normal kidney function [2,9-12]. Moreover, the primary cause of mortality in ESRD patients is also cardiovascular events [7]. Therefore, we expected that the most common cause of postoperative mortality would be adverse cardiovascular events. However, sepsis was responsible for more than $30 \%$ of the postoperative 30 -day mortality in our ESRD patients, making it the most common cause.

Several studies have reported higher infection rates in ESRD patients. After hip arthroplasty, the deep infection rate exceeded $10 \%$ in dialysis patients $[17,18]$. De la Garza Ramos et al. [19] found that the infection rate was significantly in- 
creased in patients with late-stage renal disease, who had almost 2.4 times greater odds of experiencing a surgical site infection than matched controls within 90 days after posterolateral lumbar fusion. Many orthopedic surgery studies have found that the patients with CKD or ESRD had higher surgical site infection and wound complication rates than patients with normal renal function $[19,20]$. In our study, the origin of sepsis in the mortality cases was not directly related to surgical site infection. However, surgery causes significant physiological stress and suppresses immune system activity [21-24]. Therefore, we presumed that the sepsis causing death resulted from exacerbating the immunologic vulnerability of ESRD by surgical stress. ESRD patients have immune changes due to protein-energy wasting, which means losing protein mass and energy stores due to oxidative stress, acidemia, and nutrient loss [22,23]. Furthermore, neutrophils tend to undergo apoptosis in uremic plasma conditions, especially in ESRD patients, which leads to lymphocytopenia and $\mathrm{T}$ lymphocyte function impairment $[25,26]$. CKD and ESRD patients also have more pro-inflammatory cytokine interleukin- 6 receptors due to decreased excretion than healthy controls [27]. Moreover, their fluid overload and edematous state and comorbidities like atherosclerosis and diabetes mellitus can exacerbate the pro-inflammatory condition [28]. Gupta et al. [29] found that the plasma pro-inflammatory cytokine levels and positive acute-phase proteins were higher in participants with lower kidney function and greater albuminuria. HD has also long been thought to contribute to the inflammatory response [30]. Regardless of the cause of sepsis, it is clear that ESRD patients undergoing general anesthesia are more susceptible to inflammation and infection by various routes.

Erkocak et al. [20] found that DM and a higher BMI were significantly associated with increased infection risk. Although almost $50 \%$ of our ESRD cohort had DM, the association between DM and mortality was not significant. Nevertheless, the relation between DM and infection is worthy of further research regarding ESRD patients' postoperative mortality due to inflammation [6]. Inconsistent with Erkocak et al. [20], ESRD patients with a higher BMI had low mortality $(\mathrm{OR}=0.92, \mathrm{P}=0.020)$ in our univariable analysis and BMI was not significant in the multivariable analysis. The mean BMI in our patients was lower than in Erkocak et al. [20] (23.48 vs. $\left.30.25 \mathrm{~kg} / \mathrm{m}^{2}\right)$. This is likely to reflect malnutrition and might increase mortality. This is consistent with the relationship between lower plasma albumin and higher mortality in our study $(\mathrm{OR}=0.35, \mathrm{P}<0.001)$. Further stud- ies are required to verify the association between the postoperative mortality of ESRD patients and low BMI or malnutrition, rather than obesity.

When we used the serum potassium as a confounding variable, the finding that the higher the serum potassium, the lower the risk was found to be misleading because there was little hyperkalemia in the data, because adequate serum potassium levels were achieved through preoperative dialysis. Therefore, we included hyperkalemia and hypokalemia in the analysis to address this issue, instead of serum potassium levels. Since there was almost no hyperkalemia, it was difficult to find statistical significance in hyperkalemia, while hypokalemia was significant in the univariable analysis, but not in the multivariable analysis. Further studies are needed to compare volatile anesthesia in ESRD patients, which was significant in our multivariable analysis $(\mathrm{OR}=3.21, \mathrm{P}=$ 0.032), with total intravenous anesthesia.

This study has several limitations. First, it was a single-center retrospective study and there was no control group of patients with normal renal function or not under dialysis. We cannot assure that our data represent all ESRD patients. Second, we did not consider patients with a broad range of disease severity during the period when they required dialysis, including patients with preoperative sepsis or who were immunocompromised. There might be a relationship between disease severity and postoperative mortality; further study considering a broader range of disease severity in ESRD patients is needed to verify this. Third, we did not analyze the types of surgery; certain types might have affected the patient mortality. In addition, patients who had multiple surgeries during the period might have had a much higher mortality risk, but this was not included in the analysis. Finally, we lacked laboratory data that reflect the level and severity of inflammation and infection, such as C-reactive protein.

In the general population, adverse cardiovascular events are a major cause of morbidity and mortality after non-cardiac surgery. In our study, however, more than $30 \%$ of the postoperative mortality was caused by sepsis. This suggests that immunological issues have a significant role in the death of ESRD patients after general anesthesia. However, considering the above-mentioned limitations and numerous factors affecting mortality that could not be investigated, our study has low explanatory power. Nevertheless, it could inform surgeons and anesthesiologists that it is essential to understand ESRD patients' immune-suppressed state, emphasizing the importance of preventing inflammation and infection. 


\section{FUNDING}

None.

\section{CONFLICTS OF INTEREST}

No potential conflict of interest relevant to this article was reported.

\section{DATA AVAILABILITY STATEMENT}

The datasets generated during and/or analyzed during the current study are available from the corresponding author on reasonable request.

\section{AUTHOR CONTRIBUTIONS}

Conceptualization: Sun Young Park. Data curation: Sun Young Park. Formal analysis: Jae Hwa Yoo. Methodology: Sanghoon Song, Mun Gyu Kim. Project administration: Ho Bum Cho. Writing - original draft: Chaeyeon Cho. Writing review \& editing: Sanghoon Song, Sang Ho Kim. Supervision: Ji Won Chung.

\section{ORCID}

Sanghoon Song, https://orcid.org/0000-0002-9327-2472 Chaeyeon Cho, https://orcid.org/0000-0003-1593-5696 Sun Young Park, https://orcid.org/0000-0003-2588-3324 Ho Bum Cho, https://orcid.org/0000-0001-9540-1525 Jae Hwa Yoo, https://orcid.org/0000-0003-0675-0761 Mun Gyu Kim, https://orcid.org/0000-0003-1992-8215 Ji Won Chung, https://orcid.org/0000-0003-4573-9100 Sang Ho Kim, https://orcid.org/0000-0003-3781-5353

\section{REFERENCES}

1. Khwaja A. KDIGO clinical practice guidelines for acute kidney injury. Nephron Clin Pract 2012; 120: c179-84.

2. Kanda H, Hirasaki Y, Iida T, Kanao-Kanda M, Toyama Y, Chiba $\mathrm{T}$, et al. Perioperative management of patients with end-stage renal disease. J Cardiothorac Vasc Anesth 2017; 31: 2251-67.

3. Hall JE, Mouton AJ, da Silva AA, Omoto ACM, Wang Z, Li X, et al. Obesity, kidney dysfunction, and inflammation: interactions in hypertension. Cardiovasc Res 2021; 117: 1859-76.

4. Hamrahian SM, Falkner B. Hypertension in chronic kidney disease. Adv Exp Med Biol 2017; 956: 307-25.
5. Thomas MC, Cooper ME, Zimmet P. Changing epidemiology of type 2 diabetes mellitus and associated chronic kidney disease. Nat Rev Nephrol 2016; 12: 73-81.

6. Saran R, Robinson B, Abbott KC, Bragg-Gresham J, Chen X, Gipson D, et al. US renal data system 2019 annual data report: epidemiology of kidney disease in the United States. Am J Kidney Dis 2020; 75(1 Suppl 1): A6-7.

7. Korean Society of Nephrology. 2019 Korean ESRD registry. Seoul, Korean Society of Nephrology. 2019.

8. Webster AC, Nagler EV, Morton RL, Masson P. Chronic kidney disease. Lancet 2017; 389: 1238-52.

9. Palamuthusingam D, Johnson DW, Hawley CM, Pascoe E, Sivalingam P, Fahim M. Perioperative outcomes and risk assessment in dialysis patients: current knowledge and future directions. Intern Med J 2019; 49: 702-10.

10. Mathew A, Devereaux PJ, O'Hare A, Tonelli M, Thiessen-Philbrook H, Nevis IF, et al. Chronic kidney disease and postoperative mortality: a systematic review and meta-analysis. Kidney Int 2008; 73: 1069-81.

11. Chikuda H, Yasunaga H, Horiguchi H, Takeshita K, Kawaguchi H, Matsuda S, et al. Mortality and morbidity in dialysis-dependent patients undergoing spinal surgery: analysis of a national administrative database in Japan. J Bone Joint Surg Am 2012; 94: 433-8.

12. Brakoniecki K, Tam S, Chung P, Smith M, Alfonso A, Sugiyama G. Mortality in patients with end-stage renal disease and the risk of returning to the operating room after common general surgery procedures. Am J Surg 2017; 213: 395-8.

13. Currie A, Malietzis G, Askari A, Nachiappan S, Swift P, Jenkins JT, et al. Impact of chronic kidney disease on postoperative outcome following colorectal cancer surgery. Colorectal Dis 2014; 16: 879-85.

14. Singer M, Deutschman CS, Seymour CW, Shankar-Hari M, Annane D, Bauer M, et al. The third international consensus definitions for sepsis and septic shock (sepsis-3). JAMA 2016; 315: 801-10

15. Nagayoshi Y, Kawano H, Kojima S, Soejima H, Kaikita K, Nakayama M, et al. Significance of coronary vasospasm in the perioperative management of non-cardiac surgery. Circ J 2012; 76: 1965-71

16. Briasoulis A, Bakris GL. Chronic kidney disease as a coronary artery disease risk equivalent. Curr Cardiol Rep 2013; 15: 340.

17. Lieberman JR, Fuchs MD, Haas SB, Garvin KL, Goldstock L, Gupta R, et al. Hip arthroplasty in patients with chronic renal failure. J Arthroplasty 1995; 10: 191-5.

18. Sakalkale DP, Hozack WJ, Rothman RH. Total hip arthroplasty in patients on long-term renal dialysis. J Arthroplasty 1999; 14: 
571-5.

19. De la Garza Ramos R, Jain A, Nakhla J, Nasser R, Puvanesarajah V, Hassanzadeh H, et al. Postoperative morbidity and mortality after elective anterior cervical fusion in patients with chronic and end-stage renal disease. World Neurosurg 2016; 95: 480-5.

20. Erkocak OF, Yoo JY, Restrepo C, Maltenfort MG, Parvizi J. Incidence of infection and inhospital mortality in patients with chronic renal failure after total joint arthroplasty. J Arthroplasty $2016 ; 31: 2437-441$.

21. Rosenfeldt F, Wilson M, Lee G, Kure C, Ou R, Braun L, et al. Oxidative stress in surgery in an ageing population: pathophysiology and therapy. Exp Gerontol 2013; 48: 45-54.

22. Kato S, Chmielewski M, Honda H, Pecoits-Filho R, Matsuo S, Yuzawa Y, et al. Aspects of immune dysfunction in end-stage renal disease. Clin J Am Soc Nephrol 2008; 3: 1526-33.

23. Fouque D, Kalantar-Zadeh K, Kopple J, Cano N, Chauveau P, Cuppari L, et al. A proposed nomenclature and diagnostic criteria for protein-energy wasting in acute and chronic kidney disease. Kidney Int 2008; 73: 391-8. Erratum in: Kidney Int 2008; 74: 393.

24. Ben-Eliyahu S, Page GG, Yirmiya R, Shakhar G. Evidence that stress and surgical interventions promote tumor development by suppressing natural killer cell activity. Int J Cancer 1999; 80: 880-8.
25. Cendoroglo M, Jaber BL, Balakrishnan VS, Perianayagam M, King AJ, Pereira BJ. Neutrophil apoptosis and dysfunction in uremia. J Am Soc Nephrol 1999; 10: 93-100.

26. Sela S, Shurtz-Swirski R, Cohen-Mazor M, Mazor R, Chezar J, Shapiro G, et al. Primed peripheral polymorphonuclear leukocyte: a culprit underlying chronic low-grade inflammation and systemic oxidative stress in chronic kidney disease. J Am Soc Nephrol 2005; 16: 2431-8. Erratum in: J Am Soc Nephrol 2005; 16: 2814.

27. Memoli B, Postiglione L, Cianciaruso B, Bisesti V, Cimmaruta C, Marzano L, et al. Role of different dialysis membranes in the release of interleukin-6-soluble receptor in uremic patients. Kidney Int 2000; 58: 417-24.

28. Pecoits-Filho R, Gonçalves S, Barberato SH, Bignelli A, Lindholm B, Riella MC, et al. Impact of residual renal function on volume status in chronic renal failure. Blood Purif 2004; 22: 285-92.

29. Gupta J, Mitra N, Kanetsky PA, Devaney J, Wing MR, Reilly M, et al. CRIC Study Investigators. Association between albuminuria, kidney function, and inflammatory biomarker profile in CKD in CRIC. Clin J Am Soc Nephrol 2012; 7: 1938-46.

30. Yao Q, Axelsson J, Stenvinkel P, Lindholm B. Chronic systemic inflammation in dialysis patients: an update on causes and consequences. ASAIO J 2004; 50: lii-lvii. 